\title{
A CRACK ORIGINATING FROM A SEMICIRCULAR NOTCH IN A NON HOMOGENEOUS SHEARED HALF-PLANE
}

J. N .NNADI

(Received 10 October 2007; Revision Accepted 27 March 2009)

\begin{abstract}
An interfacial crack originates from a semi circular notch in a non-homogeneous semi infinite elastic material subjected to constant shear loads of magnitudes $T_{j}, j=1,2$. Asymptotic deformation fields near the crack tip are derived in a closed form. The stress intensity factor is shown to depend on material constants except when equal and opposite tractions are applied on load sites of equal length. Our results agree with known ones.
\end{abstract}

KEY WORDS: Interfacial crack, quarter plane, semicircular notch, sheared half plane, stress intensity factor.

\section{INTRODUCTION}

The notched homogeneous elastic half plane under remote loading has been studied in Mitchel (1965), Rice (1967) and Ejike (1973). The non-homogeneous case was studied in Nnadi, (2004a) under more practicable finite surface loading. In this paper we study the elastic half plane made from two materials. Each material is a quarter plane from which a quarter circle of radius $\mathrm{c}$ is removed. The resultant figures are then perfectly bonded along their interface to form the half plane with a semicircular notch of radius $c$. A crack of length b-c emanates from the edge of the notch along the interface. The free surface of each quarter plane is subjected to anti-plane shear loads of magnitude $T_{j}$, on interval $\left[a_{j}, b_{j}\right], j=1,2$. Every other surface is stress free (fig 1). The subscript $\mathrm{j}=1$ refers to the upper quarter plane while the subscript $\mathrm{j}=2$ refers to the other material.

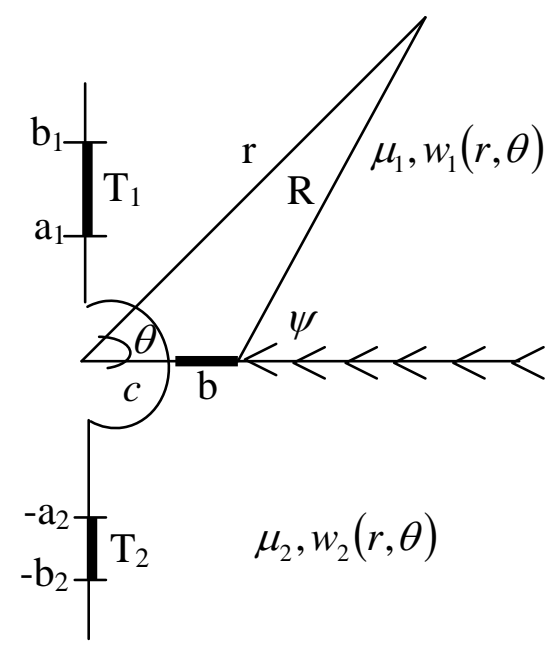

Fig. 1: Geometry Of The Problem

The polar stresses are:

$$
\sigma_{j \theta z}(r, \theta)=\frac{\mu_{j}}{r} \frac{\partial w_{j}}{\partial \theta}(r, \theta) ; \quad \sigma_{j \theta z}(r, \theta)=\mu_{j} \frac{\partial w_{j}}{\partial r}(r, \theta), j=1,2
$$

The problem is to find $\mathrm{w}_{\mathrm{j}}(r, \theta)$ in the boundary value problem: 


$$
\begin{gathered}
\left(\frac{\partial^{2}}{\partial r^{2}}+\frac{1}{r} \frac{\partial}{\partial r}+\frac{1}{r^{2}} \frac{\partial^{2}}{\partial \theta^{2}}\right) w_{j}(r, \theta)=0, r \geq c,-\frac{\pi}{2} \leq \theta \leq \frac{\pi}{2}, \quad j=1,2 \\
w_{1}(r, 0)=w_{2}(r, 0) ; \quad \mu_{1} \frac{\partial w_{1}}{\partial \theta}(r, 0)=\mu_{2} \frac{\partial w_{2}}{\partial \theta}(r, 0), \quad c \leq r \leq b \\
\frac{\partial w_{j}}{\partial \theta}\left(r, \pm \frac{\pi}{2}\right)=\frac{r T_{j}}{\mu_{j}}, \quad a_{j} \leq r \leq b_{j}, \quad j=1,2 \\
=0, \quad r<a_{j}, r>b_{j}, \quad j=1,2 \\
\frac{\partial w_{j}}{\partial \theta}(r, 0)=0, \quad c \leq r \leq b, \quad j=1,2
\end{gathered}
$$

\section{TRANSFORMATION OF THE PROBLEM}

The notched half plane is transformed on to a plane with a slit on the left half line $\operatorname{Re} \mathfrak{I}<0$ with the aid of the conformal mapping function:

$$
\mathfrak{I}(z)=\epsilon^{-2}\left(\frac{z}{c}-\frac{c}{z}\right)^{2}-1, z=x+i y, \in=\frac{b}{c}-\frac{c}{b}, b \geq c
$$

Let $\mathfrak{I}\left(r e^{i \theta}\right)=u(r, \theta)+i v(r, \theta)=\rho e^{i \phi}$ then

$$
\begin{aligned}
& u(r, \theta)=\epsilon^{-2}\left[\left(\frac{r^{2}}{c^{2}}+\frac{c^{2}}{r^{2}}\right) \cos 2 \theta-2\right]-1=\rho \cos \phi \\
& v(r, \theta)=\epsilon^{-2}\left(\frac{r^{2}}{c^{2}}-\frac{c^{2}}{r^{2}}\right) \sin 2 \theta=\rho \sin \phi
\end{aligned}
$$

Since $w_{j}(r, \theta) \equiv w_{j}(\rho, \phi)$ the displacement gradients are related through

$$
\begin{aligned}
& \frac{\partial w_{j}}{\partial \theta}(r, 0)=\frac{\partial w_{j}}{\partial \phi}(\rho, \pm \pi) \frac{\partial \phi}{\partial \theta}(r, \pm \pi / 2), a_{j} \leq r \leq b_{j}, \quad \rho>1, \quad j=1,2 \\
& \frac{\partial w_{j}}{\partial \theta}(r, 0)=\frac{\partial w_{j}}{\partial \phi}(\rho, \pm \pi) \frac{\partial \phi}{\partial \theta}(r, 0), \quad c \leq r \leq b, \rho<1, \quad j=1,2
\end{aligned}
$$

Due to the transformation the boundary value problem becomes

$$
\begin{aligned}
& \left(\frac{\partial^{2}}{\partial \rho^{2}}+\frac{1}{\rho} \frac{\partial}{\partial \rho}+\frac{1}{\rho^{2}} \frac{\partial^{2}}{\partial \phi^{2}}\right) w_{j}(\rho, \phi)=0, \rho>0,-\pi \leq \phi \leq \pi, j=1,2 \\
& w_{j}(\rho, 0)=w_{2}(\rho, 0) ; \mu_{1} \frac{\partial w_{1}}{\partial \phi}(\rho, 0)=\mu_{2} \frac{\partial w_{2}}{\partial \phi}(\rho, 0), \quad \rho>0 \\
& \frac{\partial w_{j}}{\partial \phi}(\rho, \pm \pi)=\frac{\in C}{4 \mu_{j}} \rho^{\frac{1}{2}}\left[\left(1-\rho^{-1}\right)^{-\frac{1}{2}}+\left(1-\delta \rho^{-1}\right)^{-\frac{1}{2}}\right], \alpha_{j} \leq \rho \leq \beta_{j}, j=1,2 \\
& =0 \text { otherwise }
\end{aligned}
$$

where

$$
\begin{gathered}
\delta=1+4 \epsilon^{-2} \\
\alpha_{j}=\epsilon^{-2}\left(\frac{a_{j}}{c}+\frac{c}{a_{j}}\right)^{2}+1, \beta_{j}=\epsilon^{-2}\left(\frac{b_{j}}{c}+\frac{c}{b_{j}}\right)^{2}+1
\end{gathered}
$$

\section{SOLUTION OF THE TRANSFORMED PROBLEM}

The behaviours are $w_{j}(\rho, \phi)=0\left(\rho^{\frac{1}{2}}\right)$ as $\rho \rightarrow 0$ and $w_{j}(\rho, \phi)=0\left(\rho^{-\frac{1}{2}}\right)$ as $\rho \rightarrow \infty$. The Mellin integral transform applied to (8) - (10) gives 
$\left(\frac{d^{2}}{d \phi^{2}}+s^{2}\right) \hat{w}_{j}(s, \phi)=0$

$\hat{w}_{1}(s, 0)=\hat{w}_{2}(s, 0), \mu_{1} \frac{\partial \hat{w}_{1}}{\partial \phi}(s, 0)=\mu_{2} \frac{\partial \hat{w}_{2}}{\partial \phi}(s, 0)$

$\frac{\partial \hat{w}_{j}}{\partial \phi}(s ; \pm \pi)=\frac{\in c}{4 \mu_{j}} T_{j} f\left(a_{j}, b_{j} ; s\right), j=1,2$

where the Mellin transform is defined by

$\hat{w}_{j}(s, \phi)=\int_{0}^{\infty} w_{j}(s, \phi) \rho^{s-1} d \rho,-\frac{1}{2}<\operatorname{Re} s<\frac{1}{2}, \quad j=1,2$

and

$$
f\left(a_{j}, b_{j} ; s\right)=\int_{\alpha_{j}}^{\beta_{j}} \rho^{s-1}\left[\left(1-\rho^{-1}\right)^{-\frac{1}{2}}+\left(1-\delta \rho^{-1}\right)^{-\frac{1}{2}}\right] d \rho
$$

In view of the expression Andrews (1992 p.21),

$(1-t)^{-\frac{1}{2}}=\sum_{k=0}^{\infty} c_{k} t^{k},|t|<1 ; \quad c_{k}=\frac{2 k !}{2^{2 k}(k !)^{2}}$ the integrand in (16) becomes

$\sum_{k=0}^{\infty} C_{k}\left(1+\delta^{k}\right) \rho^{s-k+\frac{1}{2}}$ and so

$f\left(a_{j}, b_{j} ; s\right)=m\left(\beta_{j} ; s\right) \beta_{j}^{s}-m\left(\alpha_{j} ; s\right) \alpha_{j}^{s}, j=1,2$

where

$$
m(t ; s)=\sum_{k=0}^{\infty} c_{k}\left(1+\delta^{k}\right) \frac{t^{-k+\frac{1}{2}}}{s-k+\frac{1}{2}}
$$

Let the solution of (12) be of the form

$\hat{w}_{j}(s, \phi)=A_{j}(s) \sin s \phi+B_{j}(s) \cos s \phi$

Application of (13) and (14) to (19) yields

$B_{1}(s)=B_{2}(s), \mu_{1} A_{1}(s)=\mu_{2} A_{2}(s)$

$A_{1}(s) \cos \pi s-B_{1}(s) \sin \pi s=\frac{\in c T_{1}}{4 \mu_{1} s} f\left(a_{1}, b_{1} ; s\right)$

$A_{2}(s) \cos \pi s-B_{2}(s) \sin \pi s=\frac{\in c T_{2}}{4 \mu_{2} s} f\left(a_{2}, b_{2} ; s\right)$

Hence

$\hat{w}_{j}(s, \phi)=\frac{\in c}{8}\left\{\left[(1+\gamma) T_{1} f\left(a_{1}, b_{1} ; s\right)+(1-\gamma) T_{2} f\left(a_{2}, b_{2} ; s\right)\right] \frac{\sin s \phi}{\mu_{j} s \cos \pi s}\right.$

$\left.+\left[(1+\gamma) \frac{T_{2}}{\mu_{2}} f\left(a_{2}, b_{2} ; s\right)-(1-\gamma) \frac{T_{1}}{\mu_{1}} f\left(a_{1}, b_{1} ; s\right)\right] \frac{\cos s \phi}{\mu_{j} s \sin \pi s}\right\}$

where Hwang et al (1994)

$\gamma=\frac{\mu_{2}-\mu_{1}}{\mu_{2}+\mu_{1}}$

The inversion formula yields the displacements as

$w_{j}(\rho, \phi)=\frac{1}{2 \pi i} \int_{a-i \infty}^{a+i \infty} \hat{w}_{j}(s, \phi) \rho^{-s} d s,-\frac{1}{2}<a<\frac{1}{2}, \quad j=1,2$ 
Residue method and Jordan's lemma, Walker (1974) are applied in seeking $w_{j}(\rho, \phi)$ for $\alpha_{j} \leq \rho \leq \beta_{j}$, by evaluating the following integrals:

$I_{\beta_{j}}^{(1)}(\rho, \phi)=\frac{1}{2 \pi i} \int_{a-i \infty}^{a+i \infty} m\left(\beta_{j} ; s\right)\left(\frac{\rho}{\beta_{j}}\right)^{-s} \frac{\sin s \phi}{s \cos \pi s} d s, \rho<\beta_{j}$

$I_{\beta_{j}}^{(2)}(\rho, \phi)=\frac{1}{2 \pi i} \int_{a-i \infty}^{a+i \infty} m\left(\beta_{j} ; s\right)\left(\frac{\rho}{\beta_{j}}\right)^{-s} \frac{\operatorname{coss} \phi}{s \sin \pi s} d s, \rho<\beta_{j}$

Note that $f\left(a_{j}, b_{j} ; s\right)=m\left(\beta_{j} ; s\right) \beta_{j}^{s}$ for $\rho \leq \beta_{j}$

$I_{\alpha_{j}}^{(1)}(\rho, \phi)=-\frac{1}{2 \pi i} \int_{a-i \infty}^{a+i \infty} m\left(\alpha_{j} ; s\right)\left(\frac{\rho}{\alpha_{j}}\right)^{-s} \frac{\operatorname{sins} \phi}{s \cos \pi s} d s, \rho<\alpha_{j}$

$I_{\alpha_{j}}^{(2)}(\rho, \phi)=-\frac{1}{2 \pi i} \int_{a-i \infty}^{a+i \infty} m\left(\alpha_{j} ; s\right)\left(\frac{\rho}{\alpha_{j}}\right)^{-s} \frac{\operatorname{coss} \phi}{s \sin \pi s} d s, \rho<\alpha_{j}$

where $f\left(a_{j}, b_{j} ; s\right)=-m\left(\alpha_{j} ; s\right) \alpha_{j}^{s}$ for $\rho \geq \alpha_{j}$

The results for $\mathrm{j}=1,2$, are:

$$
\begin{aligned}
& I_{\beta_{j}}^{(1)}(\rho, \phi)=\frac{1}{\pi i} \sum_{n=1}^{\infty} \frac{(-1)^{n-1}}{n-\frac{1}{2}} m\left(\beta_{j} ; \frac{1}{2}-n\right)\left(\frac{\rho}{\beta_{j}}\right)^{n-1 / 2} \sin (n-1 / 2) \phi \\
& +\frac{4}{\pi}\left\{-\ln \left(\frac{\rho}{\beta_{j}}\right) \sin \frac{\phi}{2}+2 \sin \frac{\phi}{2}-\phi \cos \frac{\phi}{2}\right\} \rho^{\frac{1}{2}} \\
& I_{\beta_{j}}^{(2)}(\rho, \phi)=-\frac{1}{\pi} \sum_{n=1}^{\infty} \frac{(-1)^{n}}{n} m\left(\beta_{j} ;-n\right)\left(\frac{\rho}{\beta_{j}}\right)^{n} \cos n \phi+4 \cos \frac{\phi}{2} \rho^{\frac{1}{2}} \\
& I_{\alpha_{j}}^{(1)}(\rho, \phi)=\frac{1}{\pi} \sum_{n=1}^{\infty} \sum_{k=0}^{\infty} \frac{c_{k}\left(1+\delta^{k}\right)}{(k-n)} \frac{(-1)^{n-1}}{n-1 / 2} \alpha_{j}^{n-k} \rho^{1 / 2-n} \sin (n-1 / 2) \phi \\
& +\frac{1}{\pi} \sum_{n=1}^{\infty} \frac{(-1)^{n-1}}{n-1 / 2} C_{n}\left(1+\delta^{n}\right)\left\{\ln \left(\frac{\rho}{\delta_{j}}\right) \sin (n-1 / 2) \phi-\phi \cos (n-1 / 2) \phi\right. \\
& \left.+\frac{\sin (n-1 / 2)}{n-1 / 2} \phi\right\} \rho^{1 / 2-n} \\
& I_{\alpha_{j}}^{(2)}(\rho, \phi)=\sum_{n=1}^{\infty} C_{n}\left(1+\delta^{n}\right) \rho^{1 / 2-n} \cos (n-1 / 2) \phi \\
& +\frac{1}{\pi} \sum_{n=1}^{\infty} \frac{(-1)^{n}}{n} m\left(\alpha_{j}, n\right) \alpha_{j}^{n} \rho^{-n} \cos n \phi
\end{aligned}
$$

Therefore, when $\alpha_{j}<\rho<\beta_{j}, j=1,2$, we get 


$$
\begin{aligned}
w_{j}(\rho, \phi) & =\frac{\in C}{8 \mu_{j}}\left\{(1+\gamma) T_{1}\left(I_{\beta_{j}}^{(1)}(\rho, \phi)+I_{\alpha_{j}}^{(1)}(\rho, \phi)\right)+(1-\gamma) T_{2}\left(I_{\beta_{2}}^{(1)}(\rho, \phi)+I_{\alpha_{2}}^{(1)}(\rho, \phi)\right)\right. \\
& +\mu_{j}\left[(1-\gamma) \frac{T_{2}}{\mu_{2}}\left(I_{\beta_{2}}^{(2)}(\rho, \phi)+I_{\alpha_{2}}^{(2)}(\rho, \phi)\right)\right. \\
& \left.\left.-(1-\gamma) \frac{T_{1}}{\mu_{1}}\left(I_{\beta_{1}}^{(2)}(\rho, \phi)+I_{\alpha_{1}}^{(2)}(\rho, \phi)\right)\right]\right\}
\end{aligned}
$$

For $0<\rho<\alpha_{j}$, (10a) gives $\alpha_{j}<\beta_{j}$ hence $\rho<1, \rho<\alpha_{j}$ and $\rho<\beta_{j}$ simultaneously. The integrals to be evaluated for Res $<0$ are:

$$
\begin{aligned}
I_{j}^{(1)}(\rho, \phi) & =\frac{1}{2 \pi i} \int_{a-i \infty}^{a+i \infty} f\left(a_{j}, b_{j} ; s\right) \rho^{-s} \frac{\sin s \phi}{s \cos \pi \mathrm{s}} d s \\
& =\frac{1}{\pi} \sum_{n=1}^{\infty} \frac{(-1)^{n-1}}{n-1 / 2} f\left(a_{j}, b_{j} ; 1 / 2-n\right) \rho^{n-1 / 2} \sin (n-1 / 2) \phi \\
I_{j}^{(2)}(\rho, \phi) & =\frac{1}{2 \pi i} \int_{a-i \infty}^{a+i \infty} f\left(a_{j}, b_{j} ; s\right) \rho^{-s} \frac{\cos s \phi}{s \sin \pi s} d s \\
& =\frac{1}{\pi} \sum_{n=1}^{\infty} \frac{(-1)^{n}}{n} f\left(a_{j}, b_{j} ;-n\right) \rho^{n} \cos n \phi
\end{aligned}
$$

Hence for $0<\rho<\alpha_{j}, j=1,2$

$$
\begin{aligned}
w_{j}(\rho, \phi) & =\frac{\in C}{8 \mu_{j}}\left\{(1+\gamma) T_{1} I_{1}^{(1)}(\rho, \phi)+(1+\gamma) T_{2} I_{2}^{(1)}(\rho, \phi)\right. \\
& +\mu_{j}\left[(1-\gamma) \frac{T_{2}}{\mu_{2}} I_{2}^{(2)}(\rho, \phi)-(1-\gamma) \frac{T_{1}}{\mu_{1}} I_{1}^{(2)}(\rho, \phi)\right]
\end{aligned}
$$

\section{FIELDS NEAR THE CRACK TIP}

As $\rho \rightarrow 0$ in (27) the asymptotic displacement fields near the rack tip are:

$$
\begin{aligned}
w_{j}(\rho, \phi) & =\frac{\in C}{4 \mu_{j} \pi}\left\{(1+\gamma) T_{1} f\left(a_{1}, b_{1} ;-1 / 2\right)\right. \\
& \left.+(1-\gamma) T_{2} f\left(a_{2}, b_{2} ;-1 / 2\right)\right\} \rho^{1 / 2} \sin \frac{\phi}{2}, j=1,2
\end{aligned}
$$

Consider local polar coordinates $(R, \psi)$ at the crack tip (fig 1).

Then $r \sin \theta=R \sin \psi$ and $r \cos \theta=b+R \cos \psi$. Setting $\lambda=\frac{Z}{c}-\frac{c}{Z}$ in (5a) implies $\lambda-\in \rightarrow 0$ as $z \rightarrow b$. Therefore

$$
\Im(z)=\frac{2}{\epsilon}(\lambda-\in) \text { as } z \rightarrow b
$$

Now $\lambda-\epsilon=\xi e^{i \Omega}$ implies $\xi \rightarrow 0$ as $z \rightarrow b$. That is

$$
\frac{b+\mathrm{Re}^{i \psi}}{c}-\frac{c}{b+\operatorname{Re}^{i \psi}}-\frac{b}{c}+\frac{c}{b}=\xi e^{i \Omega} \text { as } \xi \rightarrow 0
$$

Therefore $\frac{R}{c} e^{i \psi}-\frac{c}{b}\left(1-\frac{R}{b} e^{i \psi}+0\left(\frac{R}{b}\right)^{2}\right)+\frac{c}{b}=\xi e^{i \Omega}$, implies $\xi=\left(\frac{1}{c}+\frac{c}{b^{2}}\right) R$ and $\Omega=\Psi$, which applied to (29), leads to $\rho^{\frac{1}{2}} \sin \frac{\phi}{2}=\left(\frac{2}{\epsilon c}\left[1+\frac{c^{2}}{b^{2}}\right]\right)^{\frac{1}{2}} R^{\frac{1}{2}} \sin \frac{\psi}{2}$ as $\rho \rightarrow 0$ and $R \rightarrow 0$ 
Hence the crack tip displacement fields as $R \rightarrow 0$ are

$$
\begin{aligned}
w_{j}(R, \psi) & =\frac{1}{\mu_{j} \pi}\left[\frac{b}{8}\left(1-\left(\frac{c}{b}\right)^{4}\right)\right]^{\frac{1}{2}}\left[(1+\gamma) T_{1} f\left(a_{1}, b_{1} ;-1 / 2\right)\right] \\
& +(1-\gamma) T_{2} f\left(a_{2}, b_{2} ;-1 / 2\right) R^{\frac{1}{2}} \sin \frac{\psi}{2}, \quad j=1,2
\end{aligned}
$$

The conventional form of $w_{j}(R, \psi)$ is, Tada et al (1985)

$w_{j}(R, \psi)=\frac{2}{\mu} K_{I I I}\left(b-c, T_{1}, T_{2} ; \gamma_{1}\right)\left(\frac{R}{2 \pi}\right)^{\frac{1}{2}} \sin \frac{\psi}{2}$

where $b-c$ is the crack length and

$$
\begin{aligned}
K_{I I I}\left(b-c, T_{1}, T_{2} ; \gamma\right) & =\frac{1}{4}\left\{\frac{b}{\pi}\left[1-\left(\frac{c}{b}\right)^{4}\right]\right\}^{\frac{1}{2}}\left\{(1+\gamma) T_{1} f\left(a_{1}, b_{1} ;-1 / 2\right)\right. \\
& \left.+(1-\gamma) T_{2} f\left(a_{2}, b_{2} ;-1 / 2\right)\right\}
\end{aligned}
$$

is the mode III stress intensity factor.

From (17) we derive

$$
\begin{array}{r}
f\left(a_{j}, b_{j} ;-1 / 2\right)=2 \ln \frac{\beta_{j}}{\alpha_{j}}+\sum_{k=1}^{\infty} \frac{c_{k}}{k}\left(\alpha_{j}^{-k}-\beta_{j}^{-k}\right) \\
+\sum_{k=1}^{\infty} \frac{c_{k}}{k}\left[\left(\frac{\alpha_{j}}{\delta}\right)^{-k}-\left(\frac{\beta_{j}}{\delta}\right)^{-k}\right] \quad j=1,2
\end{array}
$$

Using the relation, Nnadi (2004b)

$\sum_{k=1}^{\infty} \frac{c_{k}}{k} t^{k}=-2 \ln (1+\sqrt{1-t})+2 \ln 2,|t| \leq 1$

and $(32)$ it is not difficult to apply $(11 a, b)$ to get

$$
\begin{aligned}
f\left(a_{j}, b_{j} ;-1 / 2\right)=2 \ln \{ & \left(\frac{\left.\left[\left(b_{j}+\frac{c^{2}}{b_{j}}\right)^{2}+\left(b-\frac{c^{2}}{b_{j}}\right)^{2}\right]^{\frac{1}{2}}+b_{j}+\frac{c^{2}}{b_{j}}\right)}{\left.\left[\left(a_{j}+\frac{c^{2}}{a_{j}}\right)^{2}+\left(b-\frac{c^{2}}{b}\right)^{2}\right]^{\frac{1}{2}}+a_{j}+\frac{c^{2}}{a_{j}}\right)}\right. \\
& \left(\frac{\left[\left(b_{j}+\frac{c^{2}}{b_{j}}\right)^{2}+\left(b-\frac{c^{2}}{b}\right)^{2}\right]^{\frac{1}{2}}+\left[\left(b_{j}+\frac{c^{2}}{b_{j}}\right)-4 c^{2}\right]^{\frac{1}{2}}}{\left[\left(a_{j}+\frac{c^{2}}{a_{j}}\right)^{2}+\left(b-\frac{c^{2}}{b}\right)^{2}\right]^{\frac{1}{2}}+\left[\left(a_{j}+\frac{c^{2}}{a_{j}}\right)^{2}-4 c^{2}\right]^{\frac{1}{2}}}\right)
\end{aligned}
$$

\section{CONCENTRATED SHEAR FORCE}

From the relation

$\ln \left(\frac{p}{q}\right)=\frac{p}{q}-1$ as $q \rightarrow p,\left|\frac{p}{q}-1\right|<1$, (33) becomes

$f\left(a_{j}, b_{j} ;-1 / 2\right)=2\left(b_{j}-a_{j}\right) F\left(a_{j} ;-1 / 2\right)$ as $b_{j} \rightarrow a_{j}, j=1,2$

where 


$$
F\left(a_{j} ;-1 / 2\right)=\left[1-\left(\frac{c}{a_{j}}\right)^{2}\right]\left\{\frac{\left[\left(a_{j}+\frac{c^{2}}{a_{j}}\right)^{2}-4 c^{2}\right]^{\frac{1}{2}}+a_{j}+\frac{c^{2}}{a_{j}}}{\left[\left(a_{j}+\frac{c^{2}}{a_{j}}\right)^{2}+\left(b-\frac{c^{2}}{b}\right)^{2}\right]^{\frac{1}{2}}\left[\left(a_{j}+\frac{c^{2}}{a_{j}}\right)^{2}-4 c^{2}\right]^{\frac{1}{2}}}\right\}
$$

Shear forces, $Q_{\mathrm{j}}$ concentrated at $\mathrm{a}_{\mathrm{j}}$ are achieved if $T_{j} \rightarrow \infty$ and $\left(b_{j}-a_{j}\right) T_{j} \rightarrow Q_{j}$ as $b_{j} \rightarrow a_{j}, j=1,2$. Hence $T_{j} f\left(a_{j}, b_{j} ;-1 / 2\right)=2 Q_{j} F\left(a_{j} ;-1 / 2\right)$ as $b_{j} \rightarrow a_{j}$ The intensity factor corresponding to such concentrated loading is obtained from (31) as

$$
\begin{aligned}
K_{\text {III }}\left(b-c, Q_{1}, Q_{2} ; \gamma\right) & =\frac{1}{2}\left\{\frac{b}{\pi}\left[1-\left(\frac{c}{b}\right)^{4}\right]\right\}^{\frac{1}{2}}\left\{(1+\gamma) Q_{1} F\left(a_{1}, b_{1} ;-1 / 2\right)\right. \\
& \left.+(1-\gamma) T_{2} Q_{2} F\left(a_{2} ;-1 / 2\right)\right\}
\end{aligned}
$$

\section{CONCLUSION}

If $c \neq 0$ the configuration is that of a non-inclined interfacial edge crack of depth $b$ in two bonded quarter planes Hwang et al (1994). If in addition to $c=0$, the condition $\mu_{1}=\mu_{2}$ holds, the configuration is that of an edge crack in a homogeneous half plane Nnadi (2004c). In all the cases our results agree with those for the corresponding configuration. The stress intensity factor for a load mixture of traction $T_{j}$ distributed on $\left[a_{j}, b_{j}\right], j=1$ or 2 and shear force, $Q_{i}$ concentrated at $a_{i}, i=2$ when $\mathrm{j}=1$ or $\mathrm{i}=1$ when $\mathrm{j}=2$, can be written as

$$
\begin{aligned}
K_{I I I}\left(b-c, T_{1}, Q_{2} ; \gamma\right)= & \frac{1}{4}\left\{\frac{b}{\pi}\left[1-\left(\frac{c}{b}\right)^{4}\right]\right\}^{\frac{1}{2}}\left\{(1+\gamma) T_{1} f\left(a_{1}, b_{1} ;-1 / 2\right)\right. \\
& \left.+2(1-\gamma) Q_{2} F\left(a_{2} ;-1 / 2\right)\right\} \\
K_{I I I}\left(b-c, Q_{1}, T_{2} ; \gamma\right)= & \frac{1}{4}\left\{\frac{b}{\pi}\left[1-\left(\frac{c}{b}\right)^{4}\right]\right\}^{\frac{1}{2}}\left\{2(1+\gamma) Q_{1} F\left(a_{1} ;-1 / 2\right)\right. \\
& \left.+(1-\gamma) T_{2} Q_{2} f\left(a_{2}, b_{2} ;-1 / 2\right)\right\} \\
K_{I I I}\left(b-c, T_{1}, T_{2} ; \gamma\right)= & \frac{T_{1}}{4}\left\{\frac{b}{\pi}\left[1-\left(\frac{c}{b}\right)^{4}\right]\right\}^{\frac{1}{2}}\left\{(1+\gamma) f\left(a_{1}, b_{1} ;-1 / 2\right)\right. \\
& \left.+(1-\gamma) f\left(a_{2}, b_{2} ;-1 / 2\right)\right\}, a_{1} \neq a_{2}, b_{1} \neq b_{2} \\
& =\frac{T_{1}}{2}\left(\frac{b}{\pi}\left[1-\left(\frac{c}{b}\right)^{4}\right]\right)^{\frac{1}{2}} f\left(a_{1}, b_{1} ;-\frac{1}{2}\right) a_{1}=a_{2}, b_{1}=b_{2}
\end{aligned}
$$

The result for equal and opposite concentrated loads is

$$
\begin{aligned}
K_{I I I}\left(b-c, Q_{1}, Q_{2} ; \gamma\right)= & 1 / 2 Q_{1}\left\{\frac{b}{\pi}\left[1-\left(\frac{c}{b}\right)^{4}\right]\right\}^{\frac{1}{2}}\left\{(1+\gamma) F\left(a_{1} ;-1 / 2\right)\right. \\
& \left.+(1-\gamma) F\left(a_{2} ;-1 / 2\right)\right\} \quad a_{1} \neq a_{2} \\
K_{I I I}\left(b-c, Q_{1}, Q_{2} ; \gamma\right)= & Q_{1}\left\{\frac{b}{\pi}\left[1-\left(\frac{c}{b}\right)^{4}\right]\right\}^{\frac{1}{2}} F\left(a_{1} ;-1 / 2\right), a_{1}=a_{2}
\end{aligned}
$$


These mean that dependence on material constants are suppressed when equal and opposite loads are applied at equal intervals or concentrated at points that are symmetric with respect to the crack line.

In the absence of the notch $(c=0)(35)$ gives

$F\left(a_{j} ;-1 / 2\right)=\frac{2}{\sqrt{a_{j}^{2}+b^{2}}} \quad j=1,2$

which substituted with $(36)$ (with $c=0$ ) yields the well known result [2]

$K_{I I I}\left(b, Q_{1}, Q_{2} ; \gamma\right)=\frac{(1+\gamma) Q_{1} \sqrt{b}}{\sqrt{\pi}\left(a_{1}^{2}+b^{2}\right)}+\frac{(1-\gamma) Q_{2} \sqrt{b}}{\sqrt{\pi}\left(a_{2}^{2}+b_{2}\right)}$

The graph in fig 3 shows the variation of

$K_{I I I}^{N}=K_{I I I}\left(b-c, T_{1}, T_{2}=0 ; 2 / 3\right) / \sqrt{b-c} T_{1}$ with $\frac{c}{b} c \neq 0$ for values of $\frac{c}{a_{1}}$ and $\frac{c}{b_{1}} \quad$ when

$b_{1}=2 c, a_{1}=c$ and $b_{1}=2 c, a_{1}=\frac{5}{4} c$

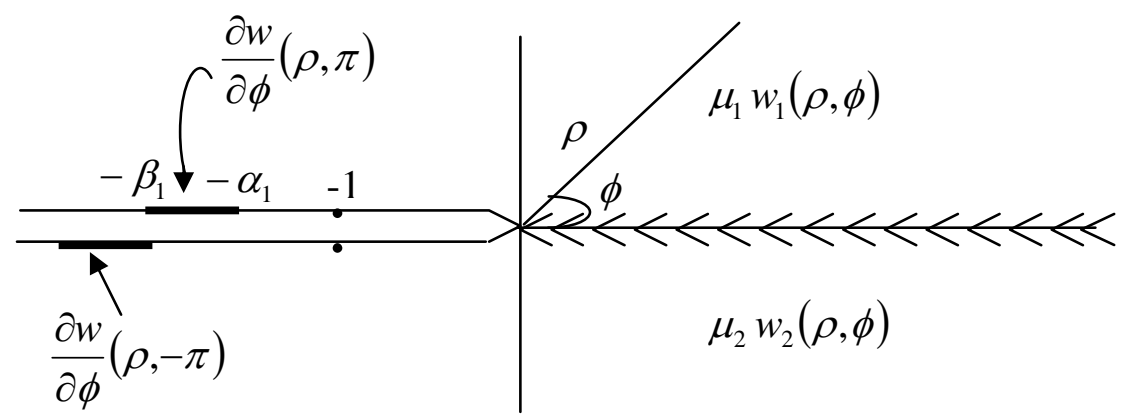

Fig. 2: Geometry of The Transformed Problem

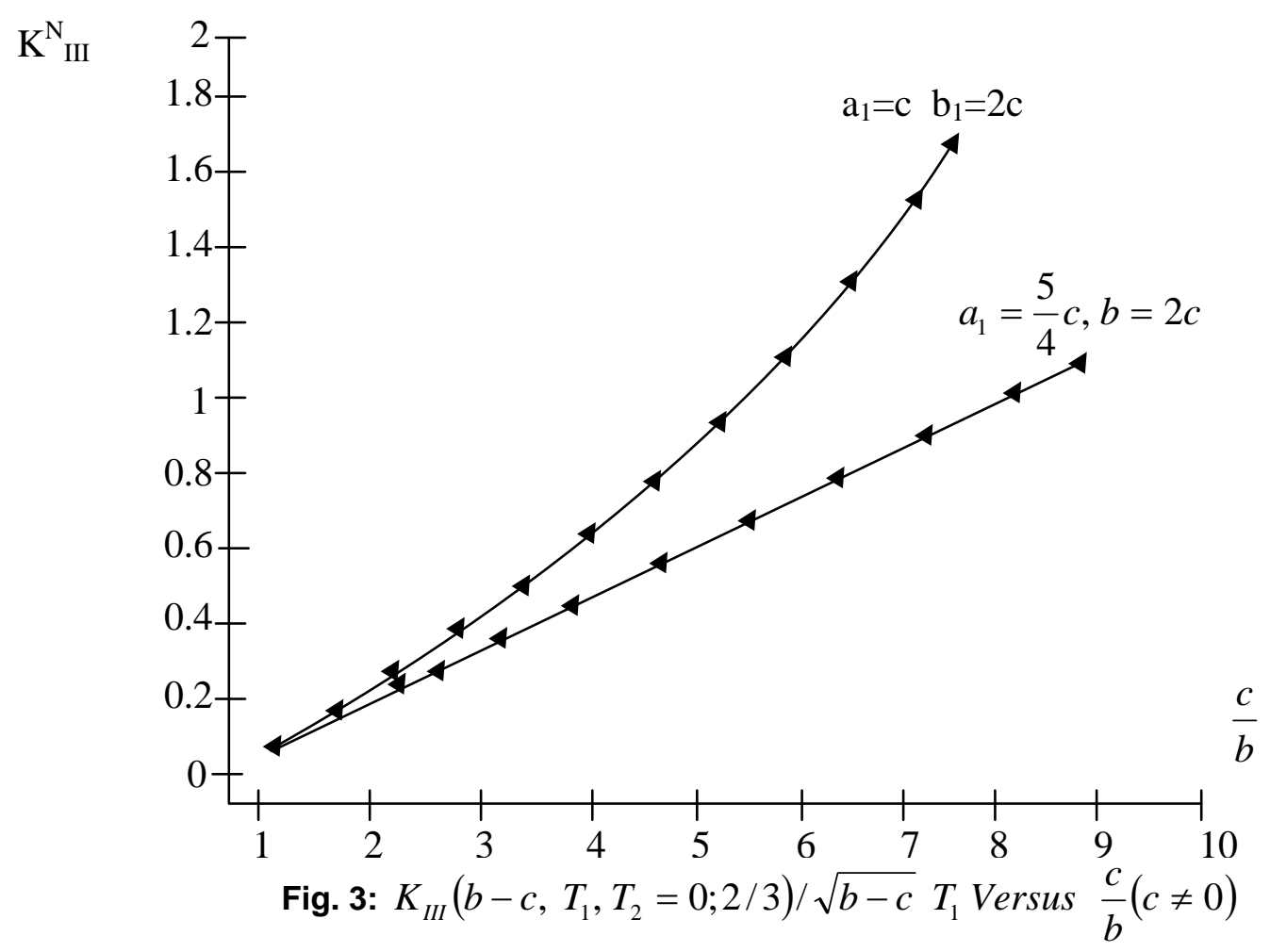




\section{REFERENCES}

Andrews, L.S., 1992. Special Functions of Mathematics for Engineers. McGraw Hill, New York.

Ejike, UBCO., 1973. Symmetrically Notched Elastic Bar. International Journal of Elasticity. Vol. 11. p. $1175-1183$

Mitchel, L.H., 1965. Stress Concentration At A Semicircular Notch. Journal of Applied Mechanics Vol 32 p. 938-939

Nnadi, J.N., 2004a Semi Circular Edge Notch In A Non Homogeneous Elastic Material. Journal of Nigerian Association of Mathematical Physics. Vol 8 p 47-56.

Nnadi, J. N., 2004b. On The Sum Of Certain Convergent Series Associated With The Beta Function. International Journal of Mathematical Education In Science And Technology Vol. 35 No 6 pp 897-941.

Nnadi, J. N., 2004c. Stress Intensity Factor For An Edge Crack In The Homogeneous Elastic Half Plane Under Variable Out-Of-Plane Loading Publications of the Institute of Mathematic and Computer Science I pp 317-328.

Rice, J. R., 1967. Stresses Due To Sharp Notched In A Work-Hardening Elastic Plastic Material Loaded By Longitudinal Shear. Journal of Applied Mechanics Vol 33 pp 287-298

Tada, H., Paris, P.C. and Irwin, G.R., 1985 Stress Analysis of Cracks Handbook Dell Research Cooperation Heller Town.

Walker, P. L., 1994. An Introduction to Complex Analysis. Adam Hilger, London. 141 pp. 